\title{
O PROBLEMA DA MOCHILA COMPARTIMENTADA E APLICAÇÕES
}

\author{
Fabiano do Prado Marques \\ Marcos Nereu Arenales * \\ Departamento de Ciências de Computação e Estatística \\ Universidade de São Paulo \\ São Carlos - SP \\ E-mails: araxa@icmc.sc.usp.br \\ arenales@icmc.sc.usp.br \\ * Corresponding author/autor para quem as correspondências devem ser encaminhadas \\ Recebido em 03/2002, aceito em 12/2002 após 2 revisões
}

\begin{abstract}
Resumo
O Problema da Mochila Compartimentada é uma variação do clássico problema da mochila e pode ser enunciado considerando-se a seguinte situação hipotética: um alpinista deve carregar sua mochila com possíveis itens de seu interesse. A cada item atribui-se o seu peso e um valor de utilidade (até aqui, o problema coincide com o clássico Problema da Mochila). Entretanto, os itens são de agrupamentos distintos (alimentos, medicamentos, utensílios, etc.) e devem estar em compartimentos separados na mochila. Os compartimentos da mochila são flexíveis e têm capacidades limitadas. A inclusão de um compartimento tem um custo fixo que depende do agrupamento com que foi preenchido, além de introduzir uma perda da capacidade da mochila. O problema consiste em determinar as capacidades adequadas de cada compartimento e como esses devem ser carregados, maximizando o valor de utilidade total, descontado o custo de incluir compartimentos. Neste trabalho propomos um modelo de otimização não linear inteiro para o problema e algumas heurísticas para sua resolução. Uma aplicação prática de relevância deste problema aparece no corte de bobinas de aço, sujeito à laminação, detalhado em apêndice.
\end{abstract}

Palavras-chave: problemas da mochila, problemas de corte e empacotamento, otimização inteira e combinatória.

\begin{abstract}
The Compartmentalized Knapsack Problem is a variation of the classical knapsack problem and can be stated considering the following hypothetical situation: an alpinist must load his knapsack with items. Two numbers, a weight and an utility value are associated with each item (so far, the problem coincides with the classical integer Knapsack Problem). However, the items are of different classes (food, medicine, utensil, etc.) and they have to be packed into separate compartments inside the knapsack. The compartments are flexible and have limited capacities. Each compartment has a fixed cost that depends on the class of the items and, in addition, each new compartment introduces a loss on the capacity of the knapsack. The Compartmentalized Knapsack Problem consists in determining suitable capacities for each compartment as well as their packing. The objective is to maximize the total utility value paid off the cost of the compartments. In this work, we model the problem as an integer non-linear optimization problem and we design some heuristic methods for its solution. A practical application of this problem arises in steel rolls cutting subject to lamination, which is discussed in detail in the appendix.
\end{abstract}

Keywords: knapsack problems, cutting and packing problems, integer and combinatorial optimization. 


\section{Introdução}

Cortar objetos grandes (por exemplo, bobinas, placas, paralelepípedos) para a produção de itens menores em quantidades bem definidos, ou empacotar itens pequenos dentro de espaços bem definidos são problemas idênticos, considerando que um item cortado de uma certa posição pode ser visto como ocupando aquela posição (daí a referência na literatura a Problemas de Corte e Empacotamento).

O número de combinações possíveis dos itens dentro de um objeto (cada combinação possível é chamada padrão de corte) é, em geral, muito grande e, a tentativa de enumerá-las completamente é inviável do ponto de vista prático. Uma função objetivo pode ser definida medindo, por exemplo, perdas no caso do problema de corte, ou vazios no caso de empacotamento, ou custos, ou número de objetos usados, etc. Um problema de corte e empacotamento consiste em determinar um padrão de corte que minimize a função objetivo.

Dentro desta categoria de problemas estão vários clássicos da literatura de pesquisa operacional, tais como os problemas da mochila, bin-packing, dentre outros, os quais são, em geral, NP-completo (Garey \& Johnson, 1979).

Vários trabalhos de revisão nos problemas de corte e empacotamento têm sido publicados nas últimas duas décadas, como Hinxman (1980), Dyckhoff et al. (1985), Dyckhoff (1990), Martello \& Toth (1990), Dowsland \& Dowsland (1992), Dyckhoff \& Finke (1992), Sweeney \& Parternoster (1992), Wäscher \& Gau (1996), Dyckhoff et al. (1997), Lodi et al. (2002) e revistas de prestígio em Pesquisa Operacional têm publicado edições especiais sobre o tema tais como Dyckhoff \& Wäscher (1990), Bischoff \& Wäscher (1995), Arenales et al. (1999).

Várias técnicas de resolução têm sido desenvolvidas, em geral especializando procedimentos consagrados da Pesquisa Operacional, tais como: enumeração implícita, programação dinâmica, relaxação lagrangeana, busca em grafos e heurísticas.

Há, na literatura, um grande número de problemas de corte e empacotamento e houve uma tentativa no trabalho de Dyckhoff (1990) de classificá-los conforme algumas características. Em particular, uma primeira característica consiste nas dimensões relevantes do processo de corte. Assim, podemos ter os problemas de corte unidimensionais, com apenas uma dimensão relevante para o processo de corte, como por exemplo, o corte de bobinas (de papel, aço, tecidos, plásticos, etc) e barras. O clássico problema da mochila pode ser visto como um problema de corte unidimensional. Outros problemas bidimensionais, onde duas dimensões são relevantes, têm aplicações diversas, como por exemplo, o corte de placas de madeira, chapas de aço, placas de vidro, tecido, etc. O problema de corte estudado neste artigo é unidimensional.

Uma outra característica dos problemas de corte e empacotamento decorre da elevada repetição de itens a serem produzidos, de modo que uma solução do problema exige o corte de vários objetos em estoque com repetição de padrões de corte (o número de objetos cortados por um mesmo padrão de corte passa a ser uma variável fundamental na formulação do problema, que é aproximado com sucesso por um problema de otimização linear). Este problema é conhecido na literatura como Problema de Corte de Estoque e os trabalhos pioneiros de Gilmore \& Gomory $(1961,1963,1965)$ utilizaram a técnica de geração de colunas e permitiram pela primeira vez que problemas práticos pudessem ser resolvidos com sucesso. A terminologia de problema de corte de estoque é também usada para problemas onde a repetição dos padrões de corte é baixa (por exemplo, apenas um objeto deve ser cortado por um padrão particular), mas há uma demanda a ser atendida, mesmo que em 
quantidade pequena (por exemplo, um ou dois itens). Neste caso, a solução arredondada da relaxação por otimização linear pode não ser uma boa aproximação. As características destacadas por Dyckhoff (1990) permitem uma classificação dos problemas de corte e empacotamento, muito embora haja uma diversidade de problemas abrigados na mesma categoria.

Técnicas de resolução de problemas de corte de estoque (sejam baseadas na geração de colunas ou heurísticas gulosas, veja Hinxman, 1980 e Wäscher \& Gau, 1996) dependem fundamentalmente da resolução de um subproblema de corte e empacotamento para a determinação do melhor padrão de corte para um particular objeto em estoque. Nesse subproblema de corte não há obrigatoriedade de satisfazer a demanda (caso a demanda seja baixa, deve-se cuidar para que a demanda não seja excedida, caso contrário, o padrão de corte gerado não seria usado uma única vez). O problema objeto deste trabalho consiste na determinação de um único padrão de corte que, como observado, é um problema fundamental na resolução de problemas mais gerais.

O Problema da Mochila Compartimentada modela a situação hipotética descrita no resumo deste trabalho com importantes aplicações práticas. Uma aplicação industrial surge no corte de bobinas de aço, sujeitas à laminação, que será mais bem explicado em apêndice.

Alguns poucos pesquisadores estudaram o problema de corte de bobinas de aço, onde ocorre o processo de laminação, porém o problema da mochila compartimentada foi evitado com auxílio de heurísticas simples. Por exemplo, Carvalho \& Rodrigues (1994) utilizam a técnica de geração de colunas, mas para a determinação dos padrões de corte (onde surge o problema da mochila compartimentada) reduzem a busca a soluções homogêneas para cada compartimento. Em Ferreira et al. (1990) uma heurística gulosa é utilizada na geração de um conjunto de padrões de corte, sem a restrição de compartimentação, para então descartar aqueles que não podem ser compartimentados através de regras heurísticas simples. Em Pereira (1993) encontra-se uma boa descrição do problema, considerando o processo de laminação e uma modelagem matemática que o autor concluiu ser ineficiente na resolução de problemas práticos. Hoto $(1996,2001)$ e Marques (2000) focalizaram seus estudos no problema da mochila compartimentada. Em particular, Hoto (1996) fornece os primeiros passos para uma modelagem e métodos heurísticos e, mais tarde Hoto (2001), apresenta métodos exatos e heurísticos para a mochila compartimentada, que difere do presente trabalho por não incluir a limitação da quantidade de itens no padrão de corte (na literatura de corte e empacotamento é chamado de problema irrestrito). Mais recentemente, Zak (2002) estudou um problema relacionado com o problema da mochila compartimentada, onde uma seqüência de estágios no processo de corte obriga que bobinas intermediárias sejam produzidas antes dos itens finais, definindo uma espécie de compartimentos. Porém, os itens podem ser acomodados livremente dentro de cada compartimento, isto é, não há uma partição dos itens em agrupamentos (medicamentos, alimentos, etc.), como suposto no problema da mochila compartimentada a ser estudado neste trabalho.

Uma variedade de problemas da mochila vem sendo estudada há várias décadas pela comunidade de Pesquisa Operacional e revisados, por exemplo, nos trabalhos de Martelo \& Toth (1990) e Lin (1998). Porém o problema da mochila compartimentada não foi estudado.

Na seção 2 definimos e modelamos o problema da mochila compartimentada. Na seção 3 propomos três métodos heurísticos e seus desempenhos computacionais são apresentados na seção 4. Por fim, em apêndice, mostramos uma aplicação prática de relevância que aparece no corte de bobinas de aço, sujeito à laminação. 


\section{O Problema da Mochila Compartimentada}

Nesta seção formalizamos o problema da mochila compartimentada e apresentamos um modelo matemático de otimização não linear inteiro. Métodos heurísticos são apresentados na seção 3 .

\subsection{Definição do Problema}

Um alpinista deve carregar sua mochila com $m$ possíveis itens de sua utilidade. A cada item $i$, o alpinista atribui um valor de utilidade $v_{i}$ e seu peso $l_{i}$. O máximo peso que o alpinista suporta em sua jornada é de $L$. Até este ponto, o problema coincide com a formulação clássica do Problema da Mochila. Porém, os itens são de agrupamentos distintos (por exemplo: agrupamento 1: alimentos, agrupamento 2: utensílios, agrupamento 3: roupas, agrupamento 4: calçados, etc.) e devem estar em compartimentos separados dentro da mochila (isto é, cada compartimento contém somente itens de um mesmo agrupamento). Os compartimentos da mochila são flexíveis, permitindo que o alpinista carregue maior peso em alimentos do que em roupas, bem como reservar vários compartimentos para alimentos. As capacidades dos compartimentos (incógnitas) são limitadas inferior e superiormente (de acordo com os agrupamentos), caso estes sejam criados, digamos por: $L_{\min }^{k}$ e $L_{\max }^{k}$ (isto é, a soma dos pesos $l_{i}$ dos itens alocados no compartimento deve ser superior ou igual a $L_{\min }^{k}$ e inferior ou igual a $L_{\max }^{k}$ ). A cada compartimento é associado um custo $c_{k}$, caso este seja preenchido com itens do agrupamento $k$ e, além disso, cada compartimento incluído na mochila produzirá uma perda da capacidade da mesma, digamos, $S$ (isto é, se, por exemplo, três compartimentos forem utilizados, então a capacidade disponível na mochila será de $L-3 S$, ao invés de $L$ ).

O Problema da Mochila Compartimentada consiste em determinar as capacidades adequadas de cada compartimento e como estes devem ser carregados de modo que o valor de utilidade total (soma dos valores de utilidade de todos os itens selecionados) seja máximo, descontando-se os custos dos compartimentos, os quais dependem dos agrupamentos com que foram preenchidos $\left(c_{k}\right)$.

\subsection{Um Modelo Matemático para o Problema da Mochila Compartimentada}

Nesta seção propomos uma modelagem matemática para o Problema da Mochila Compartimentada. Observe que a cada compartimento da mochila, tem-se associado um único agrupamento $k$ de itens, ou seja, não se admite itens de agrupamentos distintos no mesmo compartimento.

\section{Dados do problema:}

- $M=\{1, \ldots, m\}:$ conjunto dos tipos de itens;

- $l_{i}$ : peso $(K g)$ do item $i\left(l_{i}>0\right), i=1, \ldots, m$;

- $v_{i}$ : valor de utilidade do item $i\left(v_{i} \geq 0\right), i=1, \ldots, m$;

- $d_{i}$ : limite máximo de itens $i$ na mochila, $i=1, \ldots, m$;

- $K$ : número total de agrupamentos distintos;

- $A_{k}$ : agrupamento $k, k=1, \ldots, K,\left(M=A_{1} \cup A_{2} \ldots \cup A_{K}, A_{i} \cap A_{j}=\varnothing\right.$, com $\left.i \neq j\right)$; 
- $N_{k}$ : número total de possíveis compartimentos para o agrupamento $k$ (por exemplo: $A_{1}=\{1,2\}$, então $L_{11}=2 l_{1}+l_{2}, L_{2 l}=l_{1}+2 l_{2}, L_{3 l}=2 l_{1}+2 l_{2}$, etc., são duas possíveis capacidades de compartimentos para o agrupamento 1 . $L_{j k}$ referencia o j-ésimo compartimento associado ao agrupamento $k$. $\mathrm{O}$ número $N_{k}$ depende essencialmente dos itens do agrupamento $k$ );

- $c_{k}$ : custo de um compartimento de itens do agrupamento $k$, onde $c_{k} \geq 0, k=1, \ldots, K$;

- $L$ : capacidade $(K g)$ da mochila;

- $L_{\text {min }}^{k}$ : capacidade mínima $(K g)$ de cada compartimento associado ao agrupamento $A_{k}$;

- $L_{\max }^{k}$ : capacidade máxima $(K g)$ de cada compartimento associado ao agrupamento $A_{k}$ $\left(L_{\min }^{k}<L_{\max }^{k}<L\right)$;

- $S$ : perda $(K g)$ decorrente da inclusão de um novo compartimento na mochila.

Variáveis:

- $\alpha_{i j k}$ : número de itens do tipo $i$, do agrupamento $k$, no compartimento do tipo $j, i=1, \ldots, m$, $k=1, \ldots, K$ e $j=1, \ldots, N_{k}$;

- $\beta_{j k}$ : número de vezes que o compartimento (padrão) do tipo $j$ alocado com o agrupamento $k$ é repetido na mochila, $k=1, \ldots, K$ e $j=1, \ldots, N_{k}$.

Assim, o j-ésimo compartimento com itens do agrupamento $k$ tem:

- A capacidade ocupada dada por:

$$
L_{j k}=\sum_{i \in A_{k}} l_{i} \alpha_{i j k}, \quad k=1, \ldots, K \text { e } j=1, \ldots, N_{k} .
$$

- O valor de utilidade dado por:

$$
V_{j k}=\sum_{i \in A_{k}} v_{i} \alpha_{i j k}, \quad k=1, \ldots, K \text { e } j=1, \ldots, N_{k} .
$$

Um modelo matemático para o problema da mochila compartimentada pode ser escrito por:

$$
\begin{array}{ll}
\text { Maximizar } & \sum_{k=1}^{K} \sum_{j=1}^{N_{k}}\left(V_{j k}-c_{k}\right) \beta_{j k} \\
\text { Sujeito a : } \quad & V_{j k}=\sum_{i \in A_{k}} v_{i} \alpha_{i j k}, \quad k=1, \ldots, K \text { e } j=1, \ldots, N_{k} \\
& L_{j k}=\sum_{i \in A_{k}} l_{i} \alpha_{i j k}, \quad k=1, \ldots, K \text { e } j=1, \ldots, N_{k} \\
& L_{\min }^{k} \leq L_{j k}+S \leq L_{\max }^{k}, \quad k=1, \ldots, K \text { e } j=1, \ldots, N_{k} \\
& \sum_{j=1}^{N_{k}} \alpha_{i j k} \beta_{j k} \leq d_{i}, \quad i=1, \ldots, m \\
& \sum_{k=1}^{K} \sum_{j=1}^{N_{k}}\left(L_{j k}+S\right) \beta_{j k} \leq L \\
& \alpha_{i j k} \geq 0, \text { inteiro, } \quad i=1, \ldots, m, \quad k=1, \ldots, K \text { e } j=1, \ldots, N_{k} \\
& \beta_{j k} \geq 0, \text { inteiro, } \quad k=1, \ldots, K \text { e } j=1, \ldots, N_{k}
\end{array}
$$


A função objetivo (3.1), a ser maximizada, fornece o valor total de utilidade, descontado o custo de alocar os compartimentos; as restrições (3.2) e (3.3) fornecem, respectivamente, o valor de utilidade e a capacidade de cada compartimento (obviamente, estas restrições podem ser eliminadas, substituindo-as nas demais restrições); as restrições (3.4) impõem limites físicos aos compartimentos, onde uma perda $S$ deve ser considerada; as restrições (3.5) limitam o número de itens na mochila (esta restrição é importante quando um número excessivo de itens deve ser evitado, embora introduza não-linearidade nas restrições) e, por fim, a restrição (3.6) correspondente aos limites físicos da mochila. Observe que o problema (3.1-3.8) é um problema de otimização não linear inteiro.

O modelo (3.1-3.8) permite considerar um agrupamento especial de itens, chamados "itens livres", os quais podem ser incluídos na mochila sem necessidade de compartimentação. Nas heurísticas a seguir, denominamos o agrupamento $K$ como o agrupamento de itens livres. Assim, para $k=K$, a restrição (3.4) é desnecessária (ou, $L_{\min }^{K}=0$ e $L_{\max }^{K}=L$ de modo que é redundante) e $c_{K}=0$. Na aplicação em interesse, isto é, no corte de bobinas de aço sujeito à laminação, os limitantes dos compartimentos são idênticos: $L_{\min }^{k}=L_{\min }$ e $L_{\max }^{k}=L_{\max }$, exceto para o compartimento dos itens livres. Nas heurísticas propostas na próxima seção usamos esta característica, embora seja facilmente implementável quando os limitantes para os compartimentos dependem dos agrupamentos.

\section{Heurísticas para o Problema da Mochila Compartimentada}

Três heurísticas "gulosas" são propostas para a resolução do Problema da Mochila Compartimentada, considerando uma quantidade máxima de itens a serem alocados na mochila $\left(d_{i}\right.$, para $\left.i=1, \ldots, m\right)$. As heurísticas apostam que o alto custo de incluir um novo compartimento sugere que, quando criado, seja o maior possível (no caso do corte de bobinas de aço, apêndice $\mathrm{A}$, este custo corresponde à laminação que é um processo caro e demorado, justificando a aposta). A seguir, fazemos uma exposição das heurísticas propostas e na seção 4 analisamos seus desempenhos computacionais.

\subsection{Heurística de Decomposição}

Esta heurística consiste de duas fases: Na primeira fase são resolvidos $(K-1)$ Problemas da Mochila de capacidade $L_{\max }$, um para cada agrupamento (exceto o agrupamento $K$ dos itens livres), resultando nos melhores compartimentos associados aos agrupamentos. Na segunda fase, um problema da mochila clássico é resolvido, considerando os compartimentos obtidos na primeira fase como superitens (na verdade, uma combinação de itens) juntamente com os itens livres, para o carregamento da mochila.

Uma solução particular para (3.1-3.8) é construída com as seguintes características:

- Com o intuito de maximizar a função objetivo (3.1) é conveniente a construção de $V_{j k}$ grande $\mathrm{e}$, portanto, para cada agrupamento $k$, construímos apenas o melhor compartimento ( $N_{k}=1$, de modo que $j$ pode ser suprimido) maximizando (3.2) restrito a (3.3), (3.4) e (3.7) (veja problema (4.1-4.3)). O valor de $\alpha_{i k}$ ( $j$ suprimido) é determinado, isto é, para cada agrupamento $k$, temos um compartimento; 
- Com $N_{k}=1$ (apenas um compartimento por agrupamento) e $\alpha_{i k}$ já determinado: determina-se $L_{k}$ (tamanho do compartimento $k$, veja (3.3), com o índice $j$ suprimido). $\mathrm{O}$ valor de $\beta_{k}$ é determinado de modo a otimizar (3.1), sujeito às demais restrições do problema (3.1-3.8) (veja problema (5.1-5.5)).

$\mathrm{O}$ algoritmo para a resolução desta heurística é apresentado a seguir.

\section{Algoritmo:}

Passo 1: Selecionar o melhor compartimento para o agrupamento $k, k=1, \ldots, K-1$, resolvendo o seguinte Problema da Mochila, de capacidade $L_{\max }$ :

$$
\begin{aligned}
& V_{k}=\text { Maximizar } \quad \sum_{i \in A_{k}} v_{i} \alpha_{i k} \\
& \text { Sujeito a : } \quad \sum_{i \in A_{k}} l_{i} \alpha_{i k}+S \leq L_{\max } \\
& \quad 0 \leq \alpha_{i k} \leq d_{i} \text { e inteiro, para } i \in A_{k}, k=1, \ldots, K-1 .
\end{aligned}
$$

Seja $L_{k}=\sum_{i \in A_{k}} l_{i} \alpha_{i k}:$ tamanho do compartimento $k$.

(note que $\alpha_{i k}$ são fixados neste passo).

Passo 2: Resolver o seguinte problema da mochila envolvendo os compartimentos determinados no passo $1 \mathrm{e}$ os itens livres:

- $\beta_{k}$ é o número de vezes que o compartimento associado ao agrupamento $k$ é repetido na mochila,

- $\gamma_{k}$ é o número de vezes que o item livre $k$ é repetido na mochila.

$$
\begin{aligned}
\text { Maximizar } & \sum_{k=1}^{K-1}\left(V_{k}-c_{k}\right) \beta_{k}+\sum_{k \in A_{K}} v_{k} \gamma_{k} \\
\text { Sujeito a }: & \sum_{k=1}^{K-1}\left(L_{k}+S\right) \beta_{k}+\sum_{k \in A_{K}} l_{k} \gamma_{k} \leq L \\
& \alpha_{i k} \beta_{k} \leq d_{i}, i \in A_{k}, k=1, \ldots, K-1 \\
& \gamma_{k} \leq d_{k}, \quad k \in A_{K}, \\
& \alpha_{i k}, \gamma_{k} \geq 0 \text { e inteiros, } i \in A_{k}, k=1, \ldots, K-1, k \in A_{K} .
\end{aligned}
$$

\subsection{Heurística do Melhor Compartimento}

Nesta heurística, como na anterior, o melhor compartimento para cada um dos agrupamentos de itens é determinado. Temos definido, então:

$$
\begin{aligned}
L_{k}^{\prime} & =\left\{\begin{array}{l}
\left(L_{k}+S\right), \text { se } k \leq K-1 \\
l_{k}, \text { se } K-1 \leq k \leq m
\end{array}\right. \\
V_{k}^{\prime} & =\left\{\begin{array}{l}
V_{k}-c_{k}, \text { se } k \leq K-1 \\
v_{k}, \text { se } K-1 \leq k \leq m
\end{array}\right.
\end{aligned}
$$


Em seguida, escolhe-se, o compartimento de maior valor por unidade $\left(V_{k}^{\prime} / L_{k}^{\prime}\right)$, sendo então, alocado na mochila. Atualizam-se os dados e repete-se o processo até que um padrão seja gerado. Com isto é evitado o problema (5.1-5.5) do procedimento anterior. Porém, apesar de apenas um compartimento ser gerado por agrupamento, a cada passo, é possível ter diferentes compartimentos com o mesmo agrupamento, algo excluído na heurística anterior, onde era permitida apenas a repetição do melhor compartimento. $\mathrm{O}$ algoritmo é apresentado passo a passo a seguir.

\section{Algoritmo:}

Passo 1: lutil $\leftarrow L$; "lutil guarda a capacidade disponível na mochila a cada iteração"

Passo 2: Para $k=1$ a $K-1$, faça:

2.1 Se $\left(\right.$ lutil $\left.\geq L_{\max }\right)$

então $L \_$compartimento $=L_{\max }$

senão Se $\left(\right.$ lutil $\left.<L_{\min }\right)$

então L_compartimento=lutil e considera-se apenas os itens livres em (7.1-7.3),

senão $\left(L_{\min } \leq\right.$ lutil $\left.<L_{\max }\right) L_{-}$compartimento $=$lutil

Observação: se $L_{-}$compartimento $<L_{\min }$ ou se $d_{i}=0, \forall i \in A_{k}$, então $V_{k}=0, k=1, \ldots, K-1$, restando apenas os itens livres para serem alocados.

2.2 Selecionar o melhor compartimento para o agrupamento $k$ resolvendo o seguinte Problema da Mochila de capacidade L_compartimento:

$$
\begin{array}{ll}
V_{k}=\text { Maximizar } \quad \sum_{i \in A_{k}} v_{i} \alpha_{i k} \\
\text { Sujeito a : } \quad \sum_{i \in A_{k}} l_{i} \alpha_{i k}+S \leq L_{-} \text {compartimento } \\
\quad 0 \leq \alpha_{i k} \leq d_{i} \text { e inteiro, para } i \in A_{k}, k=1, \ldots, K-1 .
\end{array}
$$

\section{Passo 3:}

3.1 Escolha $k^{*}$ tal que: $\operatorname{Max}\left\{\frac{V_{k}-c_{k}}{L_{k}}, k=1, \cdots, K-1 ; \frac{v_{k}}{l_{k}}, k \in A_{K}\right\}$

3.2 Atualização:

Se $k^{*}$ em (3.1) corresponder a um compartimento

então lutil $\leftarrow$ lutil $-L_{k^{*}}$ "alocar um compartimento"

$$
d_{i} \leftarrow d_{i}-\alpha_{i k} *
$$

senão lutil $\leftarrow$ lutil $-l_{k^{*}}$ "alocar um item livre"

$$
d_{i} \leftarrow d_{i}-1
$$

3.3 Se lutil $\geq \operatorname{Min}\left\{\operatorname{Min}\left\{L_{k}, k=1, \ldots, K-1\right\}, \operatorname{Min}\left\{l_{i}, \forall i \in A_{K}\right\}\right.$, volte ao Passo 2 . 


\subsection{Heurística dos " $z$ " Melhores Compartimentos}

Esta heurística consiste basicamente na determinação dos " $z$ " melhores compartimentos associados a cada agrupamento, ou seja, nas " $z$ " melhores soluções dos Problemas da Mochila para cada agrupamento. A resolução de um problema de programação linear inteira considerando estes compartimentos como superitens, juntamente com os itens livres, determina um padrão de corte para a mochila compartimentada. O algoritmo é apresentado a seguir.

\section{Algoritmo:}

Passo 1: Selecionar os " $z$ " melhores compartimentos para o agrupamento $k$ determinando as " $z$ " melhores soluções para o seguinte Problema da Mochila de capacidade $L_{\max }$, sendo $V_{1 k} \geq V_{2 k} \geq \ldots \geq V_{z k}$ os " $z$ " melhores valores de (8.1) e suas respectivas soluções $\alpha_{i j k}$.

Para $k=1, \ldots, K-1$ e $j=1, \ldots, z$, resolva:

$$
\begin{aligned}
& V_{j k}=\text { Maximizar } \quad \sum_{i \in A_{k}} v_{i} \alpha_{i j k} \\
& \text { Sujeito a : } \quad \sum_{i \in A_{k}} l_{i} \alpha_{i j k}+S \leq L_{\max } \\
& \quad 0 \leq \alpha_{i j k} \leq d_{i} \text { e inteiro, para } i \in A_{k} .
\end{aligned}
$$

Passo 2: Resolver o seguinte problema de programação inteira envolvendo os $\left((K-l)^{*} z\right)$ compartimentos selecionados e os $\left|A_{K}\right|$ itens livres:

- $\beta_{j k}$ é o número de vezes que o compartimento $j$ para o agrupamento $k$ é repetido na mochila,

- $\gamma_{k}$ é o número de vezes que o item livre $k$ é repetido na mochila.

$$
\begin{aligned}
\text { Maximizar } & \sum_{k=1}^{K-1} \sum_{j=1}^{z}\left(V_{j k}-c_{k}\right) \beta_{j k}+\sum_{k \in A_{K}} v_{k} \gamma_{k} \\
\text { Sujeito a : } & \sum_{k=1}^{K-l} \sum_{j=1}^{z} L_{j k} \beta_{j k}+\sum_{k \in A_{K}} l_{k} \gamma_{k} \leq L-\mathrm{S} \\
& \sum_{j=1}^{z} \alpha_{i j k} \beta_{j k} \leq d_{i}, \quad i \in A_{k} \text { e } k=1, \ldots, K-1 . \\
& 0 \leq \gamma_{k} \leq d_{k}, k \in A_{K} . \\
& \beta_{j k} \geq 0, \text { inteiro, } k=1, \ldots, K-1 \text { e } j=1, \ldots, z . \\
\text { onde } L_{j k}= & \sum_{i \in A_{k}} l_{i} \alpha_{i j k}+S, \quad k=1, \ldots, K-1 .
\end{aligned}
$$

Nota: Embora a Heurística de Decomposição seja um caso particular da Heurística dos " $z$ " Melhores Compartimentos, com $z=1$, destacamo-la por resolver uma seqüência de problemas da mochila. O problema (9.1-9.5) é um problema otimização inteira com várias restrições, ao contrário de (5.1-5.5) que é um problema da mochila restrito. Para resolver os problemas que surgem nas heurísticas, propusemos algumas modificações para o método de enumeração implícita proposto por Gilmore \& Gomory (1963), as quais são resumidamente apresentadas na próxima seção. 


\subsection{Resolução das Mochilas dentro das Heurísticas}

O método de enumeração implícita proposto por Gilmore \& Gomory (1963) resolve eficientemente o problema da mochila irrestrito, nos tamanhos que ocorrem no contexto dos problemas de corte, isto é, com algumas dezenas de itens. A implementação usa a estratégia backtracking de modo que o espaço de soluções é percorrido de maneira bem simples, usando basicamente um vetor solução $\alpha=\left(\alpha_{1} \alpha_{2} \ldots \alpha_{k} 0 \ldots 0\right)$, o qual tem o número de coordenadas igual ao número de itens da mochila, sendo $\alpha_{i}$ o número de itens do tipo $i$, e $k$ o último item incluído na mochila $\left(\alpha_{k}>0\right)$. O vetor solução é suficiente para caracterizar um nó na árvore de busca, de modo que o nó anterior é caracterizado por $\alpha^{\prime}=\left(\alpha_{1} \alpha_{2} \ldots \alpha_{k}-10 \ldots 0\right)$. Regras simples para incluir novos itens na mochila (portanto, novas soluções ou novos nós na árvore de busca) são facilmente derivadas, dada a simplicidade das restrições da mochila, bem como limitantes superiores. Sempre que uma solução mais valiosa é identificada, seu valor é armazenado, descartando-se a anterior. Entretanto, os problemas da mochila que necessitamos resolver dentro de cada heurística apresentam características adicionais que podem ser incluídas no processo de busca, são elas: $i$ ) a limitação do número de itens na mochila, ii) a determinação das " $z$ " melhores soluções e, iii) restrições adicionais dadas por (9.3). A inclusão de restrições adicionais no processo de busca no método de enumeração implícita de Gilmore e Gomory é bastante utilizada, porém nem sempre publicada. Por exemplo, os autores do método em seu artigo de 1963 sugeriram que restrições simples, do tipo limitação do número de facas, podem ser incluídas no processo de busca. Morabito \& Garcia (1998) também incluíram restrições adicionais no processo de busca quando estudaram um problema prático de corte (originário de uma indústria de madeira reconstituída) onde surgiam restrições difíceis de serem descritas, mas razoavelmente simples de serem incluídas no processo de busca e anunciaram excelentes resultados. A seguir, damos uma breve descrição de como incluímos as características dos problemas da mochila que ocorrem dentro das heurísticas (seções $3.1-3.3$ ).

\subsubsection{O Problema Restrito}

Os problemas de mochilas restritas que ocorrem em (4.1-4.3), (5.1-5.5) e (7.1-7.3) foram resolvidos por uma modificação simples no algoritmo de enumeração implícita de Gilmore \& Gomory (1963). As variáveis devem obedecer à limitação na quantidade de cada item dentro da mochila. Para isto, o valor atribuído à variável associada ao item $i$ (que consiste numa busca em profundidade na árvore de decisões) é calculado por:

$$
\alpha_{i}=\min \left\{d_{i},\left(\frac{\bar{L}}{l_{i}}\right)\right\}, i=1, \ldots, m .
$$

onde $\bar{L}$ é a capacidade ociosa na mochila atualizada a cada nó percorrido na árvore de busca.

O cálculo do limitante superior, importante para a eficiência do método de enumeração implícita sofre também ligeira modificação, devido à canalização das variáveis.

\subsubsection{As " $z$ " Melhores Soluções}

Os problemas (8.1-8.3) consistem nas " $z$ " melhores soluções de um problema da mochila. Para determinar tais soluções, basta armazenar no processo de busca as " $z$ " melhores soluções, ao invés de armazenar apenas a melhor, como é feito no algoritmo de Gilmore \& 
Gomory (1963). Assim, sempre que uma nova solução é determinada, o correspondente valor da função objetivo, digamos $V$, é comparado com os " $z$ " valores previamente armazenados (por facilidade estão ordenados, por exemplo: $V_{l} \geq V_{2} \geq \ldots \geq V_{z}$ ), sendo rejeitado caso $V \leq V_{z}$, ou $V_{z}$ é descartado e o valor de $V$ armazenado ordenadamente. Observe que a nova solução gerada difere das anteriores, pois o processo de busca do algoritmo de Gilmore e Gomory evita repetições de soluções. Um método alternativo pseudo-polinomial para o cálculo das melhores soluções do problema da mochila pode ser encontrado em Yanasse et al. (2000).

\subsubsection{O Problema (9.1-9.5)}

Dentre os subproblemas que surgem nas heurísticas, o problema (9.1-9.5) é o mais complexo, pois várias restrições (9.3) são adicionadas à restrição da mochila (9.2). Procedemos analogamente ao caso restrito, introduzindo uma modificação no processo de busca do método de enumeração implícita de Gilmore \& Gomory (1963), de modo a garantir a satisfação das restrições (9.3) e (9.4). Como estamos tratando com superitens (isto é, compartimentos que armazenam itens de um mesmo agrupamento) e considerando que temos " $z$ " superitens associados a um mesmo agrupamento, surge a necessidade de um maior cuidado durante a busca para que as quantidades máximas $d_{i}, i=1, \ldots, m$, não sejam violadas (veja restrições (9.3)). Para evitar tal violação é necessário primeiramente limitar a inclusão do superitem $(j, k), j=1, \ldots, z$ e $k=1, \ldots, K-1$ (j-ésimo compartimento associado ao agrupamento $k$ obtido pelo problema (8.1-8.3)) por:

$$
\beta_{j k} \leq \text { Minimo }\left\{\left\lfloor\frac{d_{i}}{\alpha_{i j k}}\right\rfloor \text {, para todo } i \in A_{k}\right\} .
$$

Isto reduz ao caso restrito já discutido, mas não é suficiente. Por exemplo, se $\beta_{l k}>0$ e $\beta_{2 k}>0$ (isto é, o primeiro e segundo compartimentos associados ao agrupamento $k$ são carregados na mochila) e, além disso, $\alpha_{i l k}>0$ e $\alpha_{i 2 k}>0$ (isto é, o item $i$ aparece nos compartimentos 1 e 2) então o total de itens do tipo $i$ já incluídos na mochila é dado por: $\alpha_{i l k} \beta_{l k}+\alpha_{i 2 k} \beta_{2 k}$ (veja restrição (9.3)). Portanto, a quantidade de superitem do tipo 3, por exemplo, é limitada por:

$$
\beta_{3 k} \leq\left\lfloor\frac{\overline{d_{i}}}{\alpha_{i 3 k}}\right\rfloor,
$$

onde $\overline{d_{i}}=d_{i}-\alpha_{i 1 k} \beta_{1 k}-\alpha_{i 2 k} \beta_{2 k}$ é a folga da restrição (9.3). A busca é similar ao caso anterior, porém considerando tais limitações para o número de compartimentos.

\section{Resultados Computacionais}

As heurísticas apresentadas na seção 3 foram testadas para uma série de exemplos gerados aleatoriamente, classificados de acordo com as seguintes 4 características:

i) número de itens livres;

ii) comprimentos dos itens;

iii) limites sobre o número de itens dos agrupamentos e,

iv) limites sobre o número de itens livres. 
Tais características tentam simular situações observadas com dados reais. A seguir apresentaremos as características que foram consideradas para cada conjunto de dados. Alguns parâmetros foram fixados para todos os exemplos, representando dados reais. Ao invés de peso, como temos utilizado até agora, usamos comprimentos da bobina e itens.

\section{- Parâmetros fixados:}

1. Capacidade da Mochila: $L=1200 \mathrm{~mm}$;

2. Capacidades mínima e máxima para os compartimentos: $L_{\min }=154 \mathrm{~mm}$ e $L_{\max }=456 \mathrm{~mm}$.

3. Perda devido à inclusão de compartimento (imperfeições nas bordas): $S=12 \mathrm{~mm}$.

4. Número de agrupamentos gerados aleatoriamente: $K \in[3,8]$.

5. Número de itens por agrupamento gerado aleatoriamente: $\left|A_{k}\right| \in[2,6]$.

6. $v_{i}=l_{i}+\sigma, \sigma \in[1,100]$ (Obs: Na geração de colunas, cada mochila compartimentada, quando a função objetivo é minimizar a perda, terá os valores de utilidade neste formato, onde $\sigma$ é o multiplicador simplex).

7. $c_{k} \in[1,100]$.

- Parâmetros variados: (para cada parâmetro, consideramos duas possibilidades)

i) Número de Itens Livres:

1: número pequeno de itens livres: $\left|A_{K}\right| \in[1,3]$

2: número grande de itens livres: $\left|A_{K}\right| \in[4,10]$

ii) Comprimentos dos Itens:

3: Itens pequenos: $l_{i} \in[36 \mathrm{~mm}, 105 \mathrm{~mm}]$.

4: Itens grandes e pequenos: $l_{i} \in[36 \mathrm{~mm}, 444 \mathrm{~mm}]$.

(Obs: na indústria, os comprimentos dos itens variam bastante de modo que a situação 4 melhor representa a realidade; a situação 3 explora o pior caso).

iii) Limites sobre o Número de Itens dos Agrupamentos:

5: limitante baixo: $d_{i} \in[1,3], i \in A_{k}, k=1, \ldots, K-1$.

6: limitante alto: $d_{i} \in[4,15], i \in A_{k}, k=1, \ldots, K-1$.

iv) Limites sobre o Número de Itens Livres:

7: limitante baixo: $d_{i} \in[1,3], i \in A_{K}$.

8: limitante alto: $d_{i} \in[4,15], i \in A_{K}$.

$\mathrm{Na}$ prática das indústrias metalúrgicas, onde surge o Problema da Mochila Compartimentada, o percentual de itens livres é bastante variado pode ser da ordem de $60 \%$ (itens livres correspondem a itens que não passam pela laminação). Embora as heurísticas descritas na seção 3 sejam projetadas para uma única mochila, devem ser executadas em outras ocasiões onde as várias situações acima podem ocorrer (por exemplo, na técnica de geração de colunas, uma mochila compartimentada deve ser resolvida a cada iteração do método simplex ou como geradora de padrões eficientes na heurística de exaustão Hinxman, 1980), de modo que observar seus desempenhos nas características acima. 
A seguir, é feito um resumo dos resultados obtidos para vários exemplos gerados. Para cada situação combinada 20 exemplos foram gerados aleatoriamente e executados para as 3 heurísticas (Decomposição, Melhor Compartimento e " $z$ " Melhores Compartimentos com $z=2$ ).

\subsection{Resultados Obtidos}

A seguir será apresentada uma tabela que mostra os valores médios obtidos para a função objetivo nos 20 exemplos executados para cada conjunto de dados.

Tabela 1 - Valores médios da função objetivo obtidos executando as heurísticas para os conjuntos de dados.

\begin{tabular}{|l|c|c|c|c|}
\hline & $\begin{array}{c}\text { Parâmetros } \\
\text { dos Conjuntos }\end{array}$ & Decomposição & $\begin{array}{c}\text { Melhor } \\
\text { Compartimento }\end{array}$ & $\begin{array}{c}\text { "z" Melhores } \\
\text { Compartimentos }\end{array}$ \\
\hline Conjunto A & $1,3,5,7$ & 2212,50 & 2143,00 & $2298,40^{*}$ \\
\hline Conjunto B & $1,3,5,8$ & 2172,00 & 2140,30 & $2206,70^{*}$ \\
\hline Conjunto C & $1,3,6,7$ & 2400,90 & $2647,70^{*}$ & 2439,70 \\
\hline Conjunto D & $1,3,6,8$ & 2850,40 & $2895,50^{*}$ & 2875,20 \\
\hline Conjunto E & $1,4,5,7$ & 1549,30 & 1578,90 & $1679,30^{*}$ \\
\hline Conjunto F & $1,4,5,8$ & 2032,40 & 1983,60 & $2044,50^{*}$ \\
\hline Conjunto G & $1,4,6,7$ & 2474,20 & $2480,80^{*}$ & 2474,20 \\
\hline Conjunto H & $1,4,6,8$ & 2138,30 & $2193,10^{*}$ & 2180,40 \\
\hline Conjunto I & $2,3,5,7$ & 2376,40 & 2364,90 & $2412,50^{*}$ \\
\hline Conjunto J & $2,3,5,8$ & 2594,10 & 2465,80 & $2602,10^{*}$ \\
\hline Conjunto K & $2,3,6,7$ & 2779,50 & 2702,20 & $2806,20^{*}$ \\
\hline Conjunto L & $2,3,6,8$ & 2988,20 & 2937,60 & $3001,90^{*}$ \\
\hline Conjunto M & $2,4,5,7$ & 2007,10 & 1920,90 & $2038,20^{*}$ \\
\hline Conjunto N & $2,4,5,8$ & 1920,70 & 1939,20 & $2075,50^{*}$ \\
\hline Conjunto O & $2,4,6,7$ & 1949,00 & 1914,40 & $2055,80^{*}$ \\
\hline Conjunto P & $2,4,6,8$ & 2476,00 & 2319,20 & $2537,10^{*}$ \\
\hline Média Total & Todas as & $\mathbf{2 3 0 7 , 5 6}$ & $\mathbf{2 2 8 9 , 1 9}$ & $\mathbf{2 3 5 7 , 9 8}$ \\
\hline
\end{tabular}

* Melhor desempenho obtido para o conjunto de dados.

Baseado nesta tabela, o gráfico a seguir foi construído. Neste gráfico, traçamos um comparativo entre os valores médios obtidos para a função objetivo (vistos na tabela 1) considerando as heurísticas executadas. Para tal, consideramos como base os valores médios obtidos para a heurística dos " $z$ " Melhores Compartimentos (correspondendo a 100\%) e de acordo com os valores obtidos nas outras heurísticas, verificamos se eles estão acima ou abaixo dos valores base. O gráfico 1 ilustra esta comparação. 


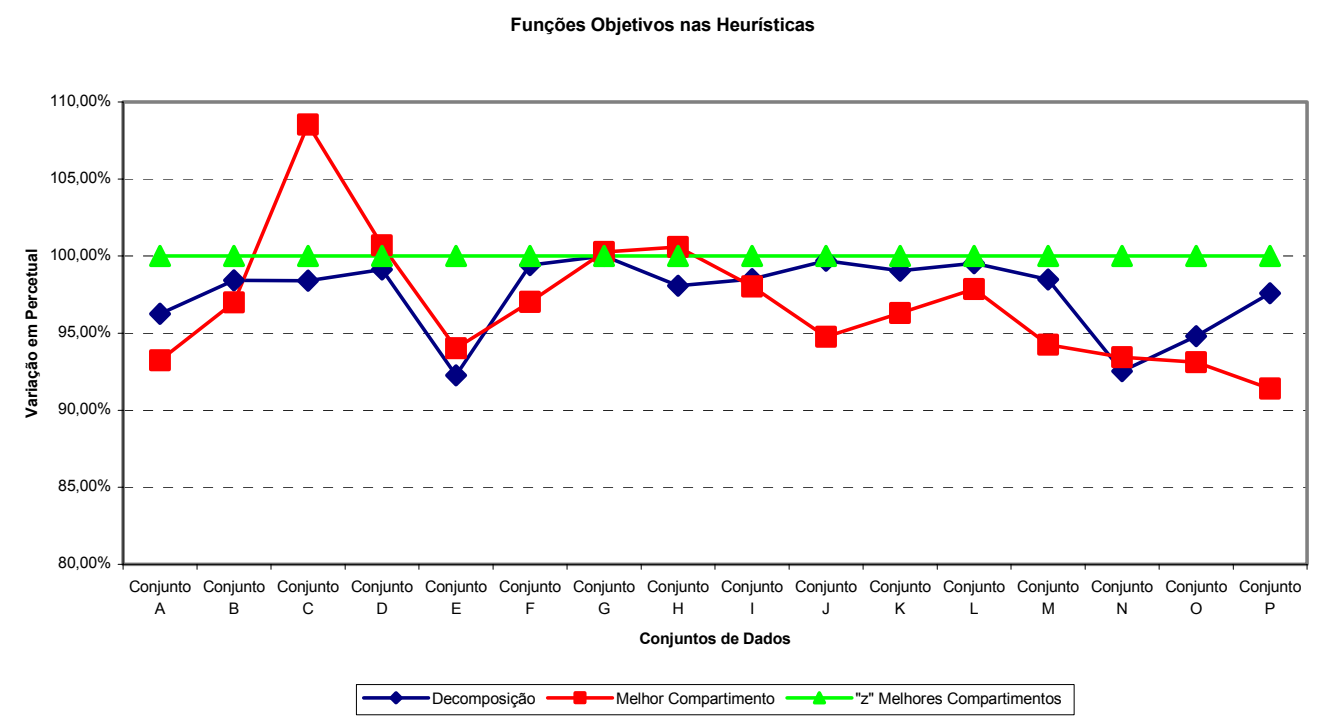

Gráfico 1 - Comparativo entre os valores obtidos para a função objetivo nas diversas heurísticas.

\subsection{Análise dos Resultados Obtidos}

A execução de vários exemplos permitiu-nos fazer uma comparação entre as três heurísticas apresentadas. Embora, de modo geral, a Heurística dos “ $z$ ” Melhores Compartimentos tenha se destacado em relação às demais, a Heurística do Melhor Compartimento apresentou resultados melhores em alguns casos específicos (conjuntos $\mathrm{C}, \mathrm{D}, \mathrm{G}, \mathrm{H}$ ). Tal situação pode ser explicada analisando as características presentes nos conjuntos de dados onde essa melhora ocorreu.

Devido à possibilidade de calcular, em seguidas iterações, compartimentos com capacidades diferentes e reduzidas, a Heurística do Melhor Compartimento não é tão dependente de muitos itens livres para gerar bons padrões de corte (exceto quando o espaço restante na mochila é inferior à capacidade mínima permitida para os compartimentos $-L_{\text {min }}$ ). Em outro aspecto, a presença de um número considerável de itens nos agrupamentos faz melhorar os valores dos compartimentos (superitens).

A Heurística dos “ $z$ " Melhores Compartimentos teve um desempenho superior na maioria dos casos, pois tal heurística permite um número maior de combinações para o preenchimento da mochila (maior número de compartimentos são gerados para cada agrupamento).

Quanto à Heurística de Decomposição, por se tratar de um caso particular da Heurística dos “ $z$ " Melhores Compartimentos (onde $z=1$ ), já era esperado que não obtivesse resultados tão destacados. Porém, ainda assim, esta heurística não perdeu tanto em desempenho para as demais, sendo, inclusive, melhor que a Heurística do Melhor Compartimento quando consideramos todos os exemplos executados para todas as combinações.

Em relação ao tempo de execução, os resultados têm mostrado que as heurísticas rodam utilizando, em média, frações de segundo e, nos piores casos, em torno de 1 segundo. Tal característica torna-se importante se desejarmos usar estas heurísticas na geração de colunas para um problema de corte de estoque (onde demandas devem ser satisfeitas). 


\section{Conclusões e Perspectivas Futuras}

Este trabalho abordou o Problema da Mochila Compartimentada, um problema de otimização combinatória, que é uma variação do clássico Problema da Mochila (porém, sua formulação matemática sofre profundas mudanças) e de importantes aplicações práticas, como no corte de bobinas de aço na indústria metalúrgica.

Um modelo matemático de otimização não linear inteiro foi proposto e nos permitiu definir um conjunto de métodos heurísticos para a sua resolução. Uma nova heurística está em desenvolvimento considerando-se a possibilidade de gerar, para cada agrupamento, " $w$ " compartimentos distintos (i.e., distintas capacidades para os compartimentos, ao invés de fixar em $L_{\max }$ ), o que melhoraria o poder combinatório para o preenchimento da mochila. Além disso, um método de geração de colunas para o problema de corte de estoque, onde demandas dos itens devem ser satisfeitas e os padrões de corte são mochilas compartimentadas vem sendo desenvolvido.

Uma outra continuação deste trabalho consiste em estender o problema aqui estudado para o caso de diversos tipos de mochilas com características diferentes (como, por exemplo, capacidades variadas). Isto levaria a considerar custos $\left(c_{k}\right)$ diferenciados de cada agrupamento a cada tipo de mochila.

Por fim, poderíamos estudar o Problema da Mochila Compartimentada para casos bidimensionais envolvendo situações encontradas na prática como, por exemplo, o problema de corte de placas de circuitos impressos (os compartimentos são sub-retângulos menores da placa original, sobre os quais incidem os mesmos processos); problemas tridimensionais, como no caso de empacotamento de caixas que devem estar agrupadas (compartimentadas) por cliente.

\section{Agradecimentos}

Os autores agradecem os vários comentários e sugestões do Prof. Dr. Robison Hoto que muito enriqueceram o trabalho. Algumas figuras deste artigo foram inspiradas ou retiradas de sua tese de doutoramento. Esta pesquisa teve apoio da FAPESP e CNPq.

\section{Referências Bibliográficas}

(1) Arenales, M.N.; Morabito, R. \& Yanasse, H. (eds.) (1999). Cutting and Packing Problems. Pesquisa Operacional, 19(2).

(2) Bischoff, E. \& Wäscher, G. (eds.) (1995). Cutting and Packing. European Journal of Operational Research, 84(3), special issue.

(3) Carvalho, J.M.V. \& Rodrigues, A.J.G. (1994). A Computer Based Interactive Approach to a Two-stage Cutting Stock Problem. INFOR, 32, 243-252.

(4) Dowsland, K.A. \& Dowsland, W.B. (1992). Packing Problems. European Journal of Operational Research, 56, 2-14.

(5) Dyckhoff, H.; Abel, D. \& Gal, T. (1985). Trim Loss and Related Problems. Omega, 13, 59-72.

(6) Dyckhoff, H. (1990). A Typology of Cutting and Packing Problems. European Journal of Operational Research, 44, 145-159. 
(7) Dyckhoff, H. \& Wäscher, G. (eds.) (1990). Cutting and Packing. European Journal of Operational Research, 44(2), special issue.

(8) Dyckhoff, H. \& Finke, U. (1992). Cutting and Packing in Production and Distribution: Typology and Bibliography. Springer-Verlag Co., Heidelberg.

(9) Dyckhoff, H.; Scheithauer, G. \& Terno, J. (1997). Cutting and Packing. Annotated Bibliographies in Combinatorial Optimization [edited by M. Amico, F. Maffioli and S. Martello], John Wiley \& Sons, New York, 393-414.

(10) Ferreira, J.S.; Neves, M.A. \& Castro, P.F. (1990). A Two-phase Roll Cutting Problem. European Journal of Operational Research, 44, 185-196.

(11) Garey, M.R. \& Johnson, D.S. (1979). Computers and Intractability: A Guide to the Theory of NP-Completeness. W.H. Freeman and Co., San Francisco.

(12) Gilmore, P. \& Gomory, R. (1961). A Linear Programming Approach to the Cutting Stock Problem. Operations Research, 9, 849-859.

(13) Gilmore, P. \& Gomory, R. (1963). A Linear Programming Approach to the Cutting Stock Problem, part II. Operations Research, 14, 94-120.

(14) Gilmore, P. \& Gomory, R. (1965). MultiStage Cutting Stock Problems os Two and More Dimensions. Operations Research, 14, 1045-1074.

(15) Hinxman, A.I. (1980). Trim-Loss and Assortment Problems: A Survey. European Journal of Operational Research, 5, 8-18.

(16) Hoto, R.S.V. (1996). Otimização no Corte de Peças Unidimensionais com Restrições de Agrupamento. Dissertação de Mestrado, ICMSC-USP, São Carlos, São Paulo.

(17) Hoto, R.S.V. (2001). O Problema da Mochila Compartimentada Aplicado no Corte de Bobinas de Aço. Tese de Doutoramento, COPPE-UFRJ, Rio de Janeiro, Rio de Janeiro.

(18) Lin, E.Y.H. (1998). A Bibliographical Survey on Some Well-Known Non-Standard Knapsack Problems. INFOR, 36(4), 274-317.

(19) Lodi, A.; Martello S. \& Monaci, M. (2002). Two-Dimensional Packing Problems: A Survey. European Journal of Operational Research, 141, 241-252,

(20) Marques, F.P. (2000). O Problema da Mochila Compartimentada. Dissertação de Mestrado, ICMC-USP, São Carlos, São Paulo.

(21) Martello, S. \& Toth, P. (1990). Knapsack Problems: Algorithms and Computer Implementations. John Wiley \& Sons, Chichester.

(22) Morabito, R. \& Arenales, M. (1992). Um exame dos problemas de corte e empacotamento. Pesquisa Operacional, 12(1), 1-20.

(23) Morabito, R. \& Garcia, V. (1998). The Cutting Stock Problem in a Hardboard Industry: A Case Study. Computers and Operations Research, 25(6), 469-485.

(24) Pereira, M.A. (1993). Uma Abordagem Matemática para o Problema de Corte e Laminação de Fitas de Aço. Dissertação de Mestrado, UNICAMP, Campinas, São Paulo.

(25) Sweeney, P. \& Paternoster, E. (1992). Cutting and Packing Problems: A Categorized, Application-Oriented Research Bibliography. Journal of the Operational Research Society, 43, 691-706. 
(26) Wäscher, G. \& Gau, T. (1996). Heuristics for the Integer One-Dimensional Cutting Stock Problem: A Computacional Study. OR Specktrum, 18, 131-144.

(27) Yanasse, H.H.; Soma, N.Y. \& Maculan, N. (2000). An algorithm for determining the K-best solutions of the one-dimensional knapsack problem. Pesquisa Operacional, 20(1), 117-134.

(28) Zak, E.J. (2002). Modeling multistage cutting stock problems. European Journal of Operational Research, 141, 313-327.

\section{Apêndice: Aplicação no Corte de Bobinas de Aço}

Descrevemos aqui o corte em bobinas de aço, sujeito à laminação, o que foi a motivação inicial deste estudo, procurando enfocar os principais aspectos envolvidos no processo. Além disso, fazemos uma descrição dos custos envolvidos no processo de produção.

Este problema foi observado em uma empresa que produz tubos de aço para diversas aplicações. A linha de produção consiste em produzir fitas, a partir de bobinas de aço em estoque. Essas fitas, por sua vez, serão utilizadas na confecção dos tubos soldados, que terão finalidades específicas.

\section{Considerações Iniciais}

\section{Terminologia:}

- Bobinas mestres são os objetos a serem cortados (mochilas a serem preenchidas). Tais bobinas são identificadas pelos seus pesos (entre 12000 e $13500 \mathrm{Kg}$ ), larguras (variando de 1100 a $1200 \mathrm{~mm}$ ), espessura do aço e pelo teor de carbono do aço segundo os critérios do SAE (Society of Automotive Engineers). Os mais utilizados são: SAE 1008, SAE 1010, SAE 1012, SAE 1017 e SAE 1021

- Bobinas intermediárias são as bobinas obtidas durante a primeira etapa de corte (os compartimentos). As bobinas intermediárias herdam algumas de suas características das bobinas mestres, como a espessura e o teor de carbono do aço. Porém, por sua vez, as bobinas intermediárias terão seus próprios pesos e larguras.

- Fitas são os itens obtidos durante a segunda etapa de corte, a partir das bobinas intermediárias. As fitas possuem características bem definidas como a largura (de acordo com o diâmetro dos tubos a serem produzidos), a espessura e o tipo de aço.

Observe que uma bobina com o tipo de aço SAE 1008 somente poderá produzir fitas com esta característica. No que se segue, supomos que o conjunto de fitas selecionado para ser produzido tenha as características necessárias de uma mesma bobina mestre a ser cortada.

\section{O Processo de Corte}

Uma peculiaridade do problema ao se efetuar cortes em bobinas de aço sujeitas a um processo técnico de laminação, reside no fato de que as bobinas mestres não podem ser laminadas devido a sua largura, pois existem restrições físicas, impostas pelo laminador, estabelecendo limites nas larguras das bobinas para que essas possam ser laminadas. Portanto, as bobinas mestres devem ser cortadas em bobinas intermediárias, cujas larguras 
respeitem as restrições do laminador e, posteriormente, recortadas em fitas. Além disso, vale enfatizar que o processo de laminação é executado visando ajustar a espessura das bobinas intermediárias de acordo com as fitas demandadas.

Sendo assim, temos duas etapas de corte bem caracterizadas, definindo um compartimento de fitas (bobinas intermediárias deverão produzir fitas de mesma espessura) na construção de um padrão de corte.

Denomina-se "Refugo" todo tipo de perda inevitável de material durante o processo de obtenção das fitas, e "Sobra" todas as outras categorias de perdas de material. Temos dois refugos intrínsecos: um durante a primeira etapa de corte e outro durante a segunda etapa de corte, ilustrado na figura 1 .

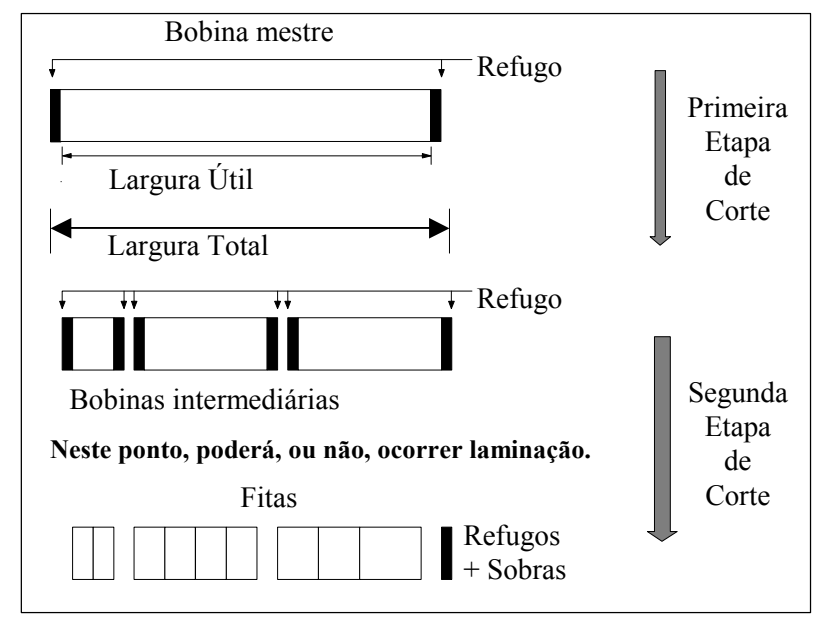

Figura 1 - Corte em bobinas de aço com refugos embutidos.

Na prática, por uma restrição no número de facas, o número de bobinas intermediárias na primeira etapa é limitado. Uma dificuldade adicional no processo de corte decorre da existência de demandas baixas, de maneira que a utilização de uma bobina mestre com peso consideravelmente alto poderá proporcionar excesso de produção, mesmo que apenas uma fita de um determinado tipo apareça no padrão de corte. Para evitar este excesso, (Hoto, 2001) considera uma formulação 1.5-dimensional, introduzindo uma variável no comprimento da bobina mestre.

\section{A Cortadeira}

Uma bobina mestre é desenrolada e o processo de corte para obtenção das bobinas intermediárias é feito longitudinalmente por "discos de corte" (não há cortes transversais e por isso podemos entender que o problema é unidimensional). Nesta etapa, existem perdas intrínsecas (que podem ser visualizadas na figura 1 como refugos) nas laterais da bobina, para eliminar as irregularidades das bordas, variando entre 6 a $8 \mathrm{~mm}$ por borda. Posteriormente, as bobinas intermediárias são enroladas e passarão, ou não, para o processo de laminação se as espessuras das fitas forem idênticas à espessura da bobina mestre, não será necessário o processo de laminação. 
O tempo de preparação da máquina de corte é cerca de 40 minutos e o tempo médio para o corte de uma bobina gira em torno de 15 minutos. A figura 2 ilustra o processo de corte.

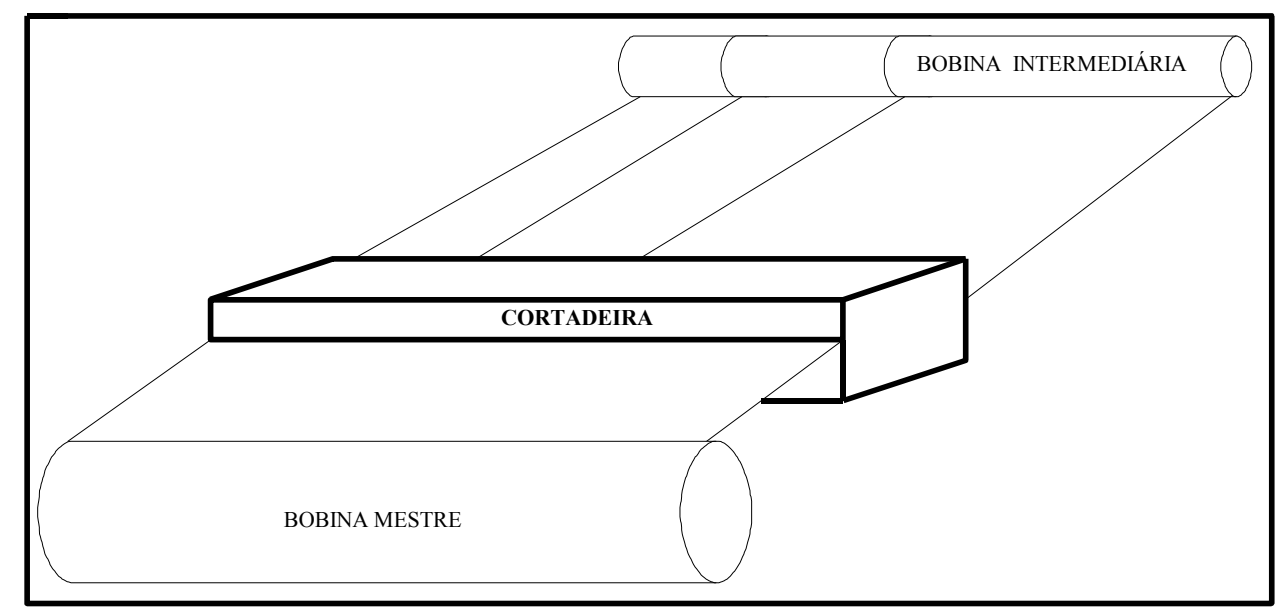

Figura 2 - Perfil do processo de corte em bobinas de aço.

\section{Laminação}

O processo de laminação tem por objetivo diminuir (quando necessário) a espessura do material de uma bobina intermediária. Esse processo se dá à temperatura ambiente (laminação a frio) por meio de cilindros de laminação que efetuam pressão sobre a lâmina de aço (figura 3). Cabe notar que, eventualmente, uma mesma bobina intermediária poderá sofrer mais de uma laminação. Isto é necessário para que se possa obter a espessura desejada do material (a redução é cerca de 15 a $20 \%$ da espessura em cada passo, e de 50 a $60 \%$ no total). Como todas as outras, a máquina que executará a laminação (o laminador) possui restrições do tipo: Não é possível laminar uma bobina intermediária com menos de $154 \mathrm{~mm}$ e mais de $456 \mathrm{~mm}$ de largura (isto limita os tamanhos dos compartimentos, conforme definição do Problema da Mochila Compartimentada, seção 2). A figura 3 ilustra o processo de laminação em perfil.

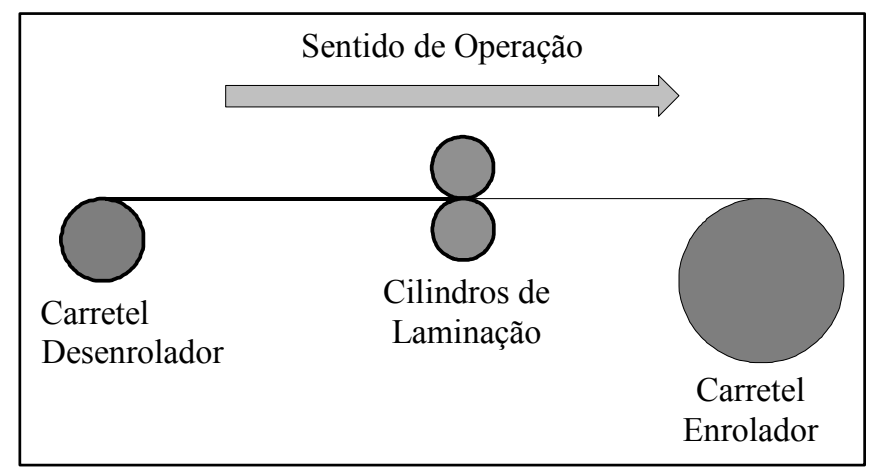

Figura 3 - Perfil do processo de laminação. 
Após a laminação, a bobina intermediária é novamente cortada para a produção das fitas. Quando uma bobina intermediária é submetida ao processo de laminação, o material adquire alguns fatores físicos indesejáveis e se faz necessário, após o recorte, um tratamento térmico ao material denominado recozimento. No recozimento, as fitas são acondicionadas em fornos especiais onde permanecem por um certo período e, em seguida, são lentamente resfriadas até atingirem a temperatura ambiente. O processo todo leva cerca de 24 horas e constitui um gargalo na produção.

Em síntese, as diversas peculiaridades da linha de produção aqui citadas apontam para um problema complexo. Além disso, a necessidade do processo de laminação induz padrões de corte pouco evidentes, pois nem todas as fitas têm a mesma espessura. Uma empresa na capital paulista que trabalha com este problema indica uma perda de material da ordem de $10 \%$, o que corresponde a uma média mensal de 500 toneladas de aço (Hoto, 2001). 